\title{
Resveratrol primes the effects of physical activity in old mice
}

\author{
Elizabeth Rodríguez-Bies ${ }^{1,2}$, Bui Thanh Tung ${ }^{1,3}$, Plácido Navas ${ }^{1}$ and Guillermo López-Lluch ${ }^{1 *}$ \\ ${ }^{1}$ Centro Andaluz de Biología del Desarrollo (CABD), Centro de Investigación Biomédica en Red de Enfermedades Raras \\ (CIBERER)-Instituto de Salud Carlos III, Universidad Pablo de Olavide-CSIC, Carretera de Utrera Km 1, 41013 Sevilla, Spain \\ ${ }^{2}$ Facultad de Ciencias del Deporte, Universidad Pablo de Olavide, Carretera de Utrera Km. 1, 41013 Sevilla, Spain \\ ${ }^{3}$ School of Medicine and Pharmacy, Vietnam National University, 144 Xuan Thuy, Cau Giay, Hanoi 10000, Vietnam \\ (Submitted 22 December 2015 - Final revision received 20 June 2016 - Accepted 5 July 2016 - First published online 4 August 2016)
}

\section{Abstract}

Decrease in muscle mass and performance with ageing is one of the main factors of frailty in the elderly. Maintenance of muscle performance by involving in physical activities is essential to increase independence and quality of life among elderly. The use of natural compounds with ergogenic activity in old people would increase the effect of moderate exercises in the maintenance of physiological muscle capacity. Resveratrol (RSV), a polyphenol found in walnuts, berries and grapes, shows this ergogenic activity. By using young, mature and old mice as models, we have found that RSV improves muscle performance in mature and old animals but not in young animals. Without showing significant effect by itself, RSV primed the effect of exercise by increasing endurance, coordination and strength in old animals. This effect was accompanied by a higher protection against oxidative damage and an increase in mitochondrial mass. RSV increased catalase and superoxide dismutase protein levels in muscle and primed exercise to reverse the decrease in their activities during ageing. Furthermore, RSV increased the level of mitochondrial mass markers such as cytochrome C, mitochondrial transcription factor A and nuclear respiratory factor-1 in muscle in exercised animals. Our results indicate that RSV can be considered an ergogenic compound that helps maintain muscle performance during ageing and subsequently reduces frailty and increases muscle performance in old individuals practising moderate exercise.

\section{Key words: Ageing: Exercise: Polyphenols: Muscles: Mitochondria: Metabolism and metabolic studies}

Sarcopenia is one of the hallmarks of ageing. This term defines age-associated loss of muscle mass, strength and function ${ }^{(1)}$. Sarcopenia is an important health problem among the elderly. It has been determined that approximately $11-50 \%$ of people aged above 80 years suffer from sarcopenia, especially those living in nursing homes ${ }^{(2)}$. Maintenance of muscle functionality is important to avoid frailty and to increase the independence and quality of life during ageing. It seems clear that for daily life activity, and hence a good quality of life, not only strength but also endurance is needed. Apart from the maintenance of a series of basic exercises, several nutritional bioactive compounds have been proposed to increase muscle function during ageing and to avoid sarcopenia ${ }^{(3)}$. These compounds act by affecting mitochondrial activity and turnover through modulating PPAR $\gamma$ co-activator $1 \alpha$ (PGC- $1 \alpha)$ and other modulators such as sirtuins ${ }^{(3)}$.

Resveratrol (RSV) is a polyphenol found in grapes, red wine, walnuts, peanuts and berries. The potential benefits of RSV have been demonstrated as a mimicker of energy intake restriction $(\mathrm{CR})^{(4)}$, modulator of postmenopausal-dependent osteopenia ${ }^{(5)}$, regulator of circadian clocks in high-fat diets ${ }^{(6)}$ or as a positive factor in motor behaviour and neuronal function during ageing ${ }^{(7)}$ among many other different effects. An enormous body of evidence indicates that RSV modulates the activity of the sirtuin- 5 ' AMP-activated protein kinase-PCG-1 $\alpha$ (SIRT-AMPK-PGC- $1 \alpha$ ) pathway and then stimulates mitochondrial turnover and biogenesis $^{(8-11)}$. This pathway is also stimulated by exercise and is responsible for a higher physical capacity and mitochondrial activity $^{(12-14)}$. Many studies performed in rodents have demonstrated the positive influence of RSV in increasing the effect of exercise on muscle ${ }^{(15-17)}$, affecting this same regulatory pathway $^{(18)}$. However, in humans, different studies carried out to determine the effect of RSV on exercise and muscle capacity have produced controversial results ${ }^{(19)}$. Dose, time and age of the individuals used in human studies can explain the differences found in the relationship with the positive effect of RSV found in rodents. In fact, the few studies performed in humans have been based on young and healthy individuals or in old individuals taking low doses of $\mathrm{RSV}^{(20-22)}$. In this study, the age reached in mice would be about 85 years in humans and the dose used in our study would represent a dairy intake amount of $1200 \mathrm{mg} /$ individual (approximately $75 \mathrm{~kg}$ ) in humans, but many of the doses used in human studies are not higher than $500-600 \mathrm{mg} / \mathrm{d}^{(20-22)}$.

Improving physical capacity during ageing is essential in order to avoid frailty and increase individual independence.

Abbreviations: CAT, catalase; CR, energy intake restriction; CytB5Rase, cytochrome $\mathrm{B}_{5}$ reductase; GPx, glutathione peroxidise; NQO1, NAD(P)H quinone dehydrogenase 1; RSV, resveratrol; SOD, superoxide dismutase.

* Corresponding author: G. López-Lluch, email glopllu@upo.es 
The design of physical exercises adapted for elderly people is very important. The incapacity of muscle to respond to physical stimuli makes the use of priming compounds important to reach a higher physical capacity and muscle performance in elderly people. The aim of this study was to determine whether RSV by itself is able to increase physical capacity or whether RSVsupplemented diets can improve the effect of training in physical performance in old organisms.

\section{Materials and methods}

\section{Animals and feeding regimens}

Male C57BL/6J mice (Charles River) were used in this study. After a week of adaptation, we started supplementation with RSV at three different ages: 2 months (young group), 12 months (mature group) and 18 months (old group) ( $n 16$ animals/ group). Animals were fed Teklad Global Diet Chow 2014S (Harlan Teklad) and housed in enriched environmental conditions in groups of 4 animals/polycarbonate cage in a colony room under a $12 \mathrm{~h}$ light $-12 \mathrm{~h}$ dark cycle (00.00-12.00 hours) under controlled temperature $\left(22 \pm 3^{\circ} \mathrm{C}\right)$ and humidity. Animals were maintained according to a protocol approved by the Ethics Committee of the University Pablo de Olavide and following the international rules for animal research.

Animals of each age group (2, 12, 18 months) were randomly divided into two groups: control and RSV. The control groups received water-containing ethanol as vehicle $(0.02 \%$ ethanol in water), whereas the RSV groups received a dose of $0 \cdot 01 \% \mathrm{RSV}$ (Cayman Chemicals) in opaque bottles to avoid light-dependent decomposition as indicated previously ${ }^{(23)}$. RSV stability was controlled in our housing conditions, and drinking water was changed twice a week for all the groups. Animals drank approximately $4-5 \mathrm{ml}$ of water/d, and their weight was approximately $30 \mathrm{~g}$ during the experiments; the calculated dose of RSV was approximately $500 \mu \mathrm{g} /$ animal per d (a calculated dose of approximately $16-17 \mathrm{mg} / \mathrm{kg}$ per $\mathrm{d}$ ).

Animals were maintained for 4.5 months under these conditions. Subsequently, mice of each group were randomly divided again into two new groups: non-trained (sedentary) and trained ( $\mathrm{T}$ ) animals. Trained animals were adapted to exercise for 1 week, and after that a training protocol was carried out using a rodent treadmill (Treadmill Columbus 1055M-E50; Cibertec SA) with $8 \%$ inclination for $20 \mathrm{~min} / \mathrm{d}$, $5 \mathrm{~d} /$ week for 6 weeks. The training protocol comprised a 3-min warm-up, followed by a training bout in which the running speed was gradually increased to $20 \mathrm{~m} / \mathrm{min}$.

At the end of the experimental procedures, ages of the animals were 8 (young group), 18 (mature group) and 24 (old group) months. After $3 \mathrm{~d}$ of the last training session or strenuous test procedure, animals were killed by cervical dislocation and the gastrocnemius muscle was quickly removed, immediately frozen in liquid $\mathrm{N}_{2}$ and stored at $-80^{\circ} \mathrm{C}$ until processing and analysis.

\section{Physical capacity determinations}

Motor activity and coordination tests were carried out on rotarod. Animals were placed on the rod until reaching $45 \mathrm{rpm}$, and the time until animals fell down was determined. Muscular force tests were performed using a Grip Strength Columbus for mice (Cibertec SA). Extenuating activity test was performed on a treadmill (Treadmill Columbus 1055M-E50; Cibertec SA) with $8 \%$ inclination starting at $10 \mathrm{~m} / \mathrm{min}$ and increasing the speed by $5 \mathrm{~m} / \mathrm{min}$ every $5 \mathrm{~min}$ until reaching $25 \mathrm{~m} / \mathrm{min}$. We established the end of the experiment as the moment the animal stopped for more than $5 \mathrm{~s}$ under electric stimuli without trying to move back to the treadmill.

\section{Tissue homogenisation}

For enzymatic and Western blot analyses, frozen gastrocnemius was homogenised in nine volumes of ice-cold, tissue-lysis buffer containing 2 mм-Tris-HCl, 20 mм-hepes, 1 mм-EDTA, $70 \mathrm{~mm}$-sucrose, $220 \mathrm{~mm}$-mannitol and $1 \mathrm{~mm}$-PMSF with protease inhibitors (Roche). Homogenates were centrifuged at $1000 \boldsymbol{g}$ for $10 \mathrm{~min}$ at $4^{\circ} \mathrm{C}$. Pellets containing unlysed cells and cellular debris were discarded. For lipid peroxidation procedures, frozen tissue was homogenised in nine volumes of ice-cold tissue-lysis buffer containing $20 \mathrm{~mm}$-Tris-HCl. Protein concentration was determined by Bradford's method.

\section{Oxidative damage assays}

Lipid peroxidation assay was performed by determining the reaction of malondialdehyde with two molecules of 1-methyl-2phenylindole at $45^{\circ} \mathrm{C}$ as indicated previously ${ }^{(23)}$. Peroxidised lipids are shown as $\mu \mathrm{mol}$ malondialdehyde equivalents/mg protein.

\section{Antioxidant activities determination}

For total superoxide dismutase (SOD) activity, the SOD determination kit (Sigma) was used as indicated by the manufacturer. Catalase (CAT) activity was measured in triplicate according to the method of Aebi ${ }^{(24)}$ by monitoring the disappearance of $\mathrm{H}_{2} \mathrm{O}_{2}$ at $240 \mathrm{~nm}$; activity was determined as nmol of hydrogen peroxide converted/min per mg total protein. Glutathione peroxidase (GPx) activity was measured using a coupled enzyme assay ${ }^{(25)}$. NAD(P)H quinone dehydrogenase 1 (NQO1) activity was determined spectrophotometrically by monitoring the reduction of the standard electron acceptor, 2,6-dichlorophenol-indophenol (DCPIP) at $600 \mathrm{~nm}^{(26)}$ in the absence or presence of dicoumarol. The dicoumarol-inhibitable part of DCPIP's reduction was calculated as NQO1 activity using the extinction coefficient of $21.0 \mathrm{~mm}^{-1} \mathrm{~cm}^{-1}$ and expressed as nmol of DCPIP reduced/min per $\mathrm{mg}$ protein. Total cytochrome $\mathrm{B}_{5}$ reductase (CytB ${ }_{5}$ Rase) activity was assayed by measuring the rate of potassium ferricyanide reduction spectrophotometrically. The enzyme activity was calculated using the extinction coefficient of $6.22 \mathrm{~mm}^{-1} \mathrm{~cm}^{-1}$ for NADH and expressed as $\mathrm{nmol} / \mathrm{min}$ per $\mathrm{mg}$ protein.

\section{Western blot analysis}

Equal amounts of protein homogenates were separated on PAGE-SDS gel and transferred onto a nitrocellulose membrane. Ponceau S (Sigma) staining was recorded using ChemiDoc ${ }^{\mathrm{TM}}$ XRS+System and compiled using Image Lab ${ }^{\mathrm{TM}}$ 4.0.1 software (Bio-Rad Laboratories) to monitor transfer efficiency and quantification of whole protein loading. Primary antibodies 
anti-CAT (219010; Calbiochem-Merck Millipore), anti-Cu, Zn-SOD (574597; Calbiochem-Merck Millipore), anti-CytB 5 Rase (rabbit polyclonal antibody kindly provided by Dr J. M. Villalba, Universidad de Córdoba, Spain) ${ }^{(27)}$, anti-cytochrome C (556433; BD Pharmingen), anti-NQO1 (C-19 sc-16464; Santa Cruz), anti-nuclear respiratory factor-1 (NRF1) (H-300 sc-33771; Santa Cruz) and anti-mitochondrial transcription factor A (mtTFAM) (A-17 sc-23588; Santa Cruz) were used. After washing, HRP-conjugated secondary antibodies were used (Calbiochem), and finally blots were developed using an enhanced chemiluminescence detection substrate Immobilon ${ }^{\mathrm{TM}}$ Western Chemiluminescent HRP Substrate (Merck Millipore). Protein levels were visualised using ChemiDoc ${ }^{\mathrm{TM}}$ XRS+System and compiled using Image $\mathrm{Lab}^{\mathrm{TM}}$ 4.0.1 software for quantification. Protein expression levels were corrected for whole protein loading determined by staining the membrane with Ponceau red $S$ and further quantification ${ }^{(28)}$.

\section{Statistical analysis}

SigmaStat 3.5 program was used for the statistical analysis and figures were obtained using SigmaPlot 10.0 program (Systat Software Inc.). All data are expressed as mean values with their standard errors. The information obtained from each group was statistically processed according to the most suitable technique for each case. Student's $t$ test and one-way or two-way ANOVA followed by post hoc pairwise multiple comparison procedures (Bonferroni's $t$ test) were performed. The critical significance level $\alpha$ was $=0.05$, and statistical significance was defined as $P<0.05$.

\section{Results}

\section{Resveratrol increases physical capacity in trained mature} and old mice

To determine whether animals fed RSV or/and trained showed different mass at the end of the experiment, we weighted them every $15 \mathrm{~d}$. Only the young group showed a significant increase during the 6 months of the procedure, whereas neither mature nor old mice showed any modification along the experiment. In each age group, trained and/or RSV-supplemented animals showed the same average weight at the end of the experiment, when the physical tests were performed, indicating no influence of mass differences between groups on their respective physical capacity (Table 1).

Treadmill tests demonstrated an age-dependent decrease in the capacity of animals to run until exhaustion, with old animals showing lower capacity to cover lesser distances, $P<0 \cdot 001$, and running for less time $P<0.001$ (Fig. 1). Mature animals also showed lower performance compared with young animals affecting both distance $(P=0.008)$ and time running until exhaustion $(P=0 \cdot 025)$. When we considered the effects of RSV and/or training in each age group, neither RSV nor training induced any improvement in the young group. However, in mature animals, RSV by itself showed a small, although not significant, increase in the distance covered, whereas training significantly increased both distance $(P=0.003)$ and time ( $P=0.010)$. Interestingly, RSV primed the effect of training, as trained animals supplemented with RSV ran until exhaustion for

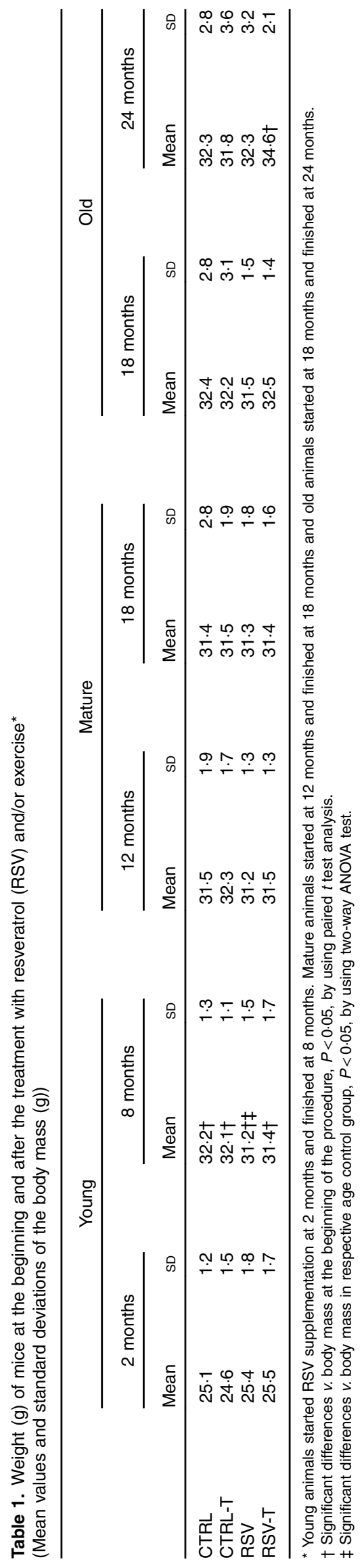


(A)

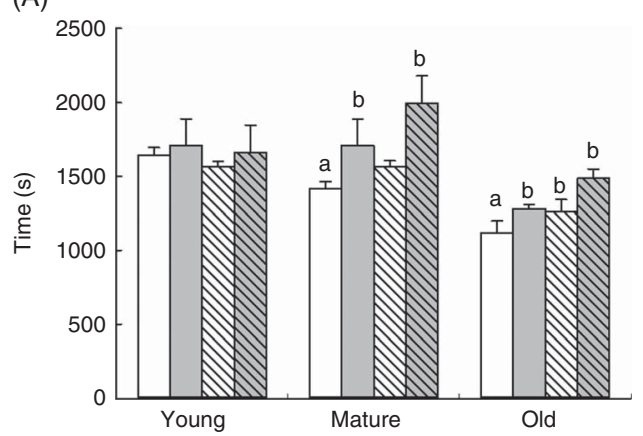

(B)

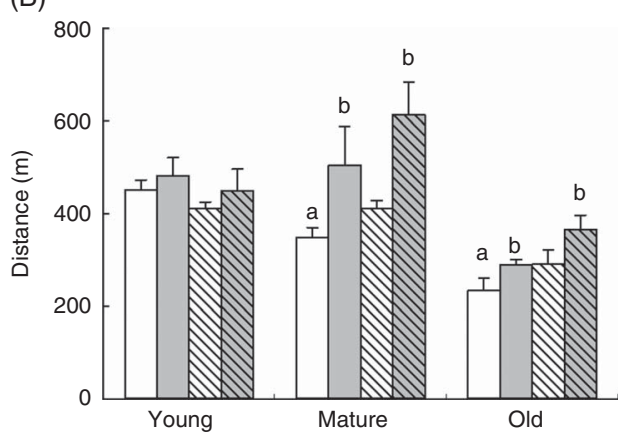

Fig. 1. Endurance performance of mice at different ages in an extenuating activity on treadmill. Endurance capacity of the animals is indicated as time after reaching extenuation (A) and the distance covered by the animals (B). Data from control animals are indicated in plain columns ( $\square$, $\square$ ). Data from resveratrol (RSV)-treated animals are indicated with dashed columns (, $\mathbb{N}$ ). Non-trained animals are shown as white columns ( $\square, \mathbb{N}$ ), and trained animals are indicated as grey columns $(\square, \mathbb{S})$. Values are means and standard deviations of the time in seconds consumed until extenuation and distance in metres covered by the animals until reaching extenuation are represented by vertical bars. ${ }^{a}$ Significant difference $v$. young control group is indicated, $P<0.05$, by using one-way ANOVA with Bonferroni's post hoc test; ${ }^{b}$ significant differences $v$. control group in each age group, $P<0.05$, by using two-way ANOVA test.

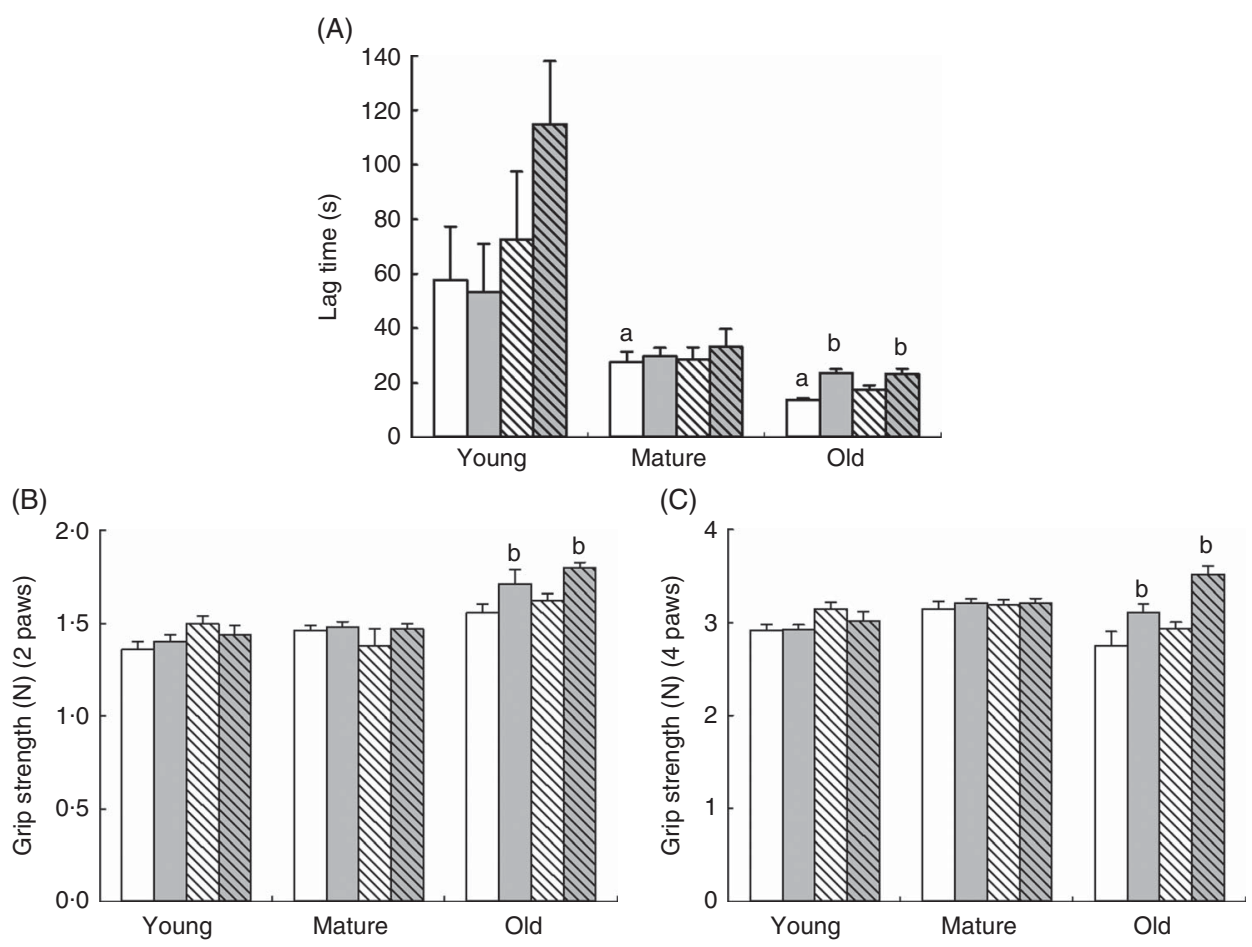

Fig. 2. Determination of coordination on rotarod (A) and grip strength (B, C) of mice at different ages. (A) Lag time (s) to fall from the rotarod and standard deviation of young, mature and old animals fed resveratrol (RSV) and/or trained. a Significant difference $v$. young control group, $P<0.05$, by using one-way ANOVA with Bonferroni's post hoc test; ${ }^{\mathrm{b}}$ significant differences $v$. control group in each age group, $P<0.05$, by using two-way ANOVA test. (B) Grip strength of the forelimbs (two paws) (B) and fore/hind limbs (four paws) (C) determined in Newtons (N) and standard deviations. ${ }^{\mathrm{b}}$ Significant differences $v$. control group in each age group, $P<0.05$,

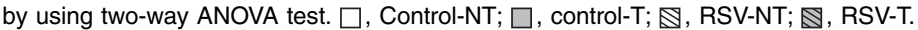

more time $(P=0.003)$ and covered more distance $(P=0.029)$. This priming effect was maintained in old animals as RSVtreated and trained animals showed the highest performance in this age group, whereas training or RSV alone improved their capacity to a lower degree. Old animals supplemented with RSV and/or trained reached similar performance as mature and young non-supplemented and non-trained animals running for the same time and covering the same distance (Fig. 1).
Rotarod experiments also demonstrated an age-dependent effect of RSV and training. Clearly, the latency to fall in the rotarod test decreased with age, already affecting mature animals. Older animals were those that maintained less time on the rod $(P=0.001)$ in comparison with control young animals (Fig. 2(a)). In this case, only old animals showed a significant increase in the latency to fall after training, $P=0 \cdot 040$, whereas young and mature animals did not show any improvement, 
although a non-significant trend to increase was found in RSVsupplemented and trained animals. Interestingly, only training improved the performance in old animals in both trained and RSV-supplemented and trained animals, $P=0.011$, and no priming effect of RSV was found in this case (Fig. 2(a)).

When muscle strength was determined by grip strength tests, the effect of RSV or exercise was found again only in old animals affecting neither young nor mature animals. We did not find significant differences in strength because of the age of animals in control groups. Training alone improved strength in both upper $(P=0.009)$ and all limbs strength $(P=0.015)$. RSV-treated and trained animals showed greater increase in strength affecting both upper limbs and forelimbs, $P=0.001$ in both cases (Fig. 2(b)).

In comparison with previous studies in mice ${ }^{(10,11)}$, our results demonstrate that low amounts of RSV show low effects on muscle performance but are able to prime the effect of exercise and affect mature and old animals more than young animals.

\section{Resveratrol reduces lipid peroxidation in old gastrocnemius muscle}

In order to study the mechanisms involved in this priming effect, we determined the degree of oxidative damage in the gastrocnemius muscle in old animals. The presence of oxidised lipids was the highest in old muscle, $P=0.001$ (Fig 3). A small decrease was found in the mature group in comparison with young animals but without reaching statistical significance. Neither exercise nor RSV produced any effect in young or mature animals. However, in the old group, RSV reduced the degree of lipid peroxidation in the gastrocnemius muscle significantly, $P=0.005$ (Fig. 3). On the other hand, training tended to increase lipid peroxidation levels in both control and RSV-supplemented animals. This tendency can be due to the longer strenuous activity performed by these animals in comparison with sedentary or non-trained animals (Fig. 1). In any case, RSV-supplemented animals showed the lowest peroxidation levels in the old group (Fig. 3).

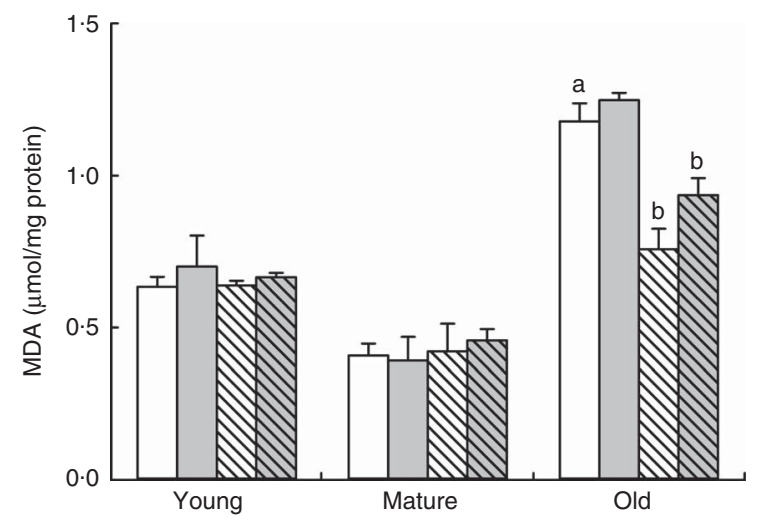

Fig. 3. Lipid peroxidation levels in different age groups fed resveratrol and/or trained. Data represent malondialdehyde (MDA) levels in muscle in $\mu \mathrm{mol} / \mathrm{mg}$ protein with their standard errors in young, mature and old animals fed resveratrol (RSV) and/or trained are represented by vertical bars. ${ }^{a}$ Significant difference $v$. young control group is indicated, $P<0.05$, by using one-way ANOVA with Bonferroni's post hoc test. ${ }^{b}$ Significant differences $v$. control group in each age group, $P<0.05$, by using two-way ANOVA test. $\square$, Control-NT; $\square$, control-T; $\mathbb{N}$, RSV-NT; $\mathbb{N}$, RSV-T.
Antioxidant activities are affected by ageing and modulated by resveratrol and physical activity

We also determined the levels of main antioxidant activities involved in general reactive oxygen species scavenging (CAT, GPX and SOD) and membrane peroxidation protection (CytB 5 Rase and NQO1) in muscle with ageing (Fig. 4). Interestingly, CAT activity decreased during ageing in muscle, $P=0.020$. Neither SOD nor GPx activities were affected. CytB5Rase and NQO1 showed a different behaviour, with CytB5Rase showing higher levels in old muscle, $P=0.034$, and NQO1 showing lower levels, $P=0.039$ (Fig. 4).

In old animals, training and RSV induced different responses in these activities. Both training and RSV showed a trend for increased CAT and SOD activities, although not reaching statistical significance. However, SOD activity was higher in animals fed RSV and trained (Fig. 5). GPx activity was not affected. In the case of $\mathrm{CytB}_{5}$ Rase, training showed a trend to increase the activity in both control and RSV-treated animals, whereas NQO1 did not respond to these effectors.

Interestingly, these low modifications in the activities were accompanied by significant changes at the protein level (Fig. 6). CAT protein levels were increased by training, RSV and their combination, $P=0.007, P=0.019, P=0.032$, respectively. Similar response was found with $\mathrm{Cu} / \mathrm{Zn} \mathrm{SOD}$ with significant increases, $P=0.026, P=0.001, P=0.001$, respectively. In the case of CAT, the effect of RSV was similar to that of training but higher in the case of SOD. No priming effect of RSV was found in these cases. No changes in the levels of $\mathrm{CytB}_{5}$ Rase protein were found, whereas NQO1 was induced only by $37 \%$ by RSV, $P=0.037$, but, interestingly, this induction was inhibited by training.

These results indicate that the lower oxidative damage found in the gastrocnemius muscle in old animals supplemented with RSV can be due, at least in part, by the induction of endogenous antioxidant defences.

\section{Mitochondrial biogenesis is enhanced by both, resveratrol and exercise}

It is known that $\mathrm{CR}$ and exercise induce mitochondrial biogenesis and then maintain higher muscle capacity ${ }^{(29)}$. In order to determine whether higher mitochondria biogenesis can be related to the higher capacity found in old animals fed RSV and trained, three different markers of mitochondrial amount were determined by Western blotting. Interestingly, cytochrome C levels were significantly higher in both RSV, $P=0.03$, and trained animals, $P=0.006$. The highest levels were found in trained animals supplemented with RSV, $P<0 \cdot 001$ (Fig. 7(a)). mtTFAM is another marker of mitochondrial levels, and in this case RSV showed the highest effect reaching three times the levels found in control animals, whereas training doubled these levels, $P=0.002$ and $P=0.049$, respectively. The combination of RSV and exercise did not increase the levels already reached with RSV, $P=0.003$ (Fig. 7(b)). Finally, the mitochondria regulator NRF1 was only induced when both RSV and exercise were combined, $P=0.008$ (Fig. 7(c)). These results indicate that the combination of RSV and training induced mitochondrial biogenesis in the gastrocnemius muscle of old animals. 
(a)

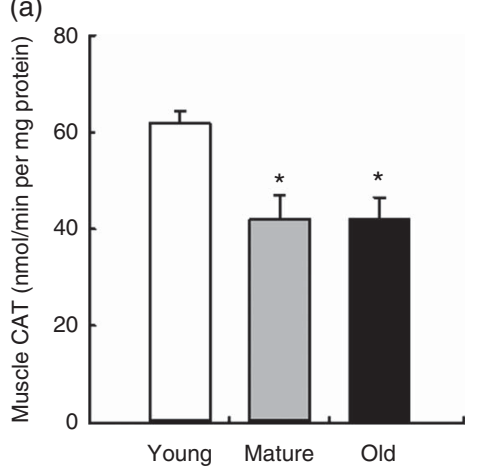

(d) (b)

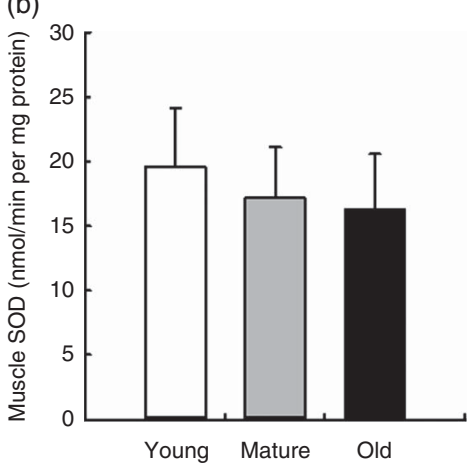

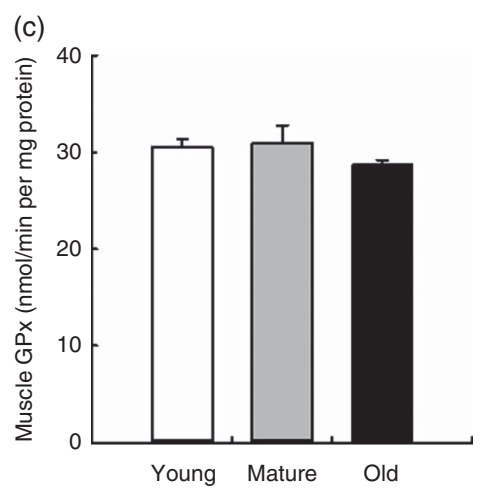

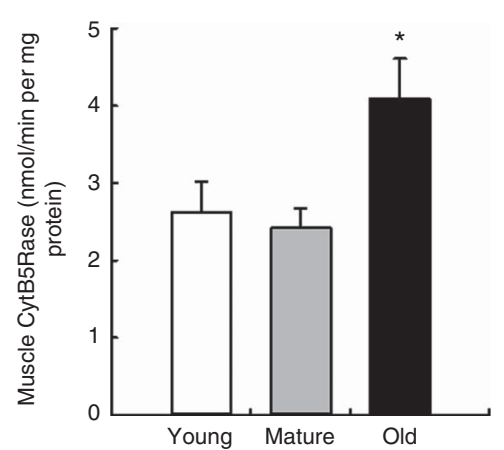

(e)

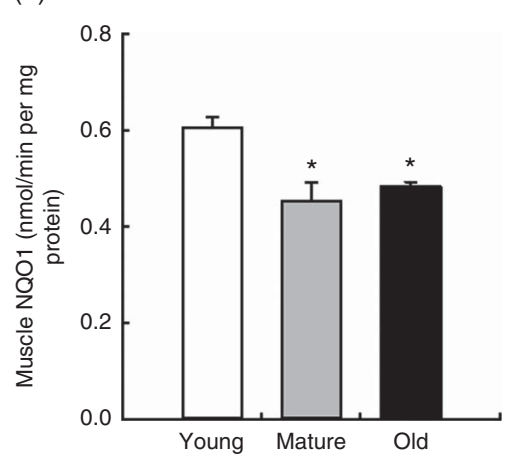

Fig. 4. Antioxidant activities in mice muscle during ageing. Values are means with their standard errors of different endogenous antioxidant activities in muscle in young, mature and old control animals are represented by vertical bars. (a) Catalase (CAT), (b) superoxide dismutase (SOD), (c) glutathione peroxidise (GPx), (d) cytochrome $B_{5}$ reductase $\left(\mathrm{CytB}_{5}\right.$ Rase) and (e) $\mathrm{NAD}(\mathrm{P}) \mathrm{H}$ quinone dehydrogenase 1 (NQO1) activities measured in nmol/min per mg protein. * Significant differences $v$. young group, $P<0.05$ by using one-way ANOVA with Bonferroni's post hoc test.

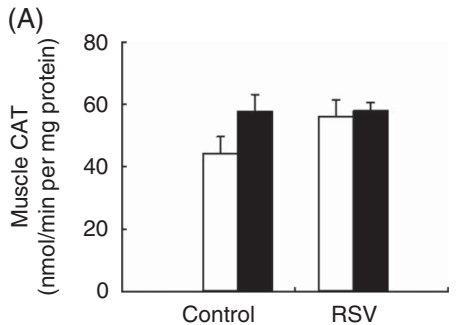

(D)

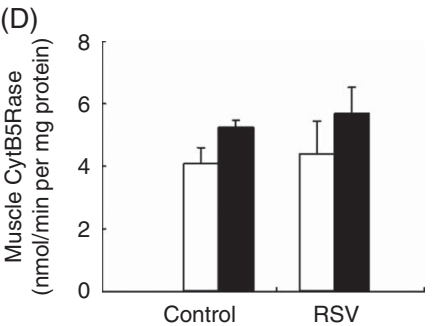

(B)

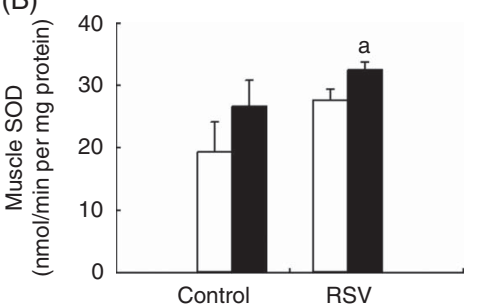

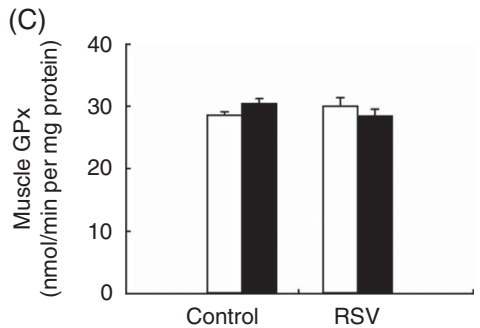

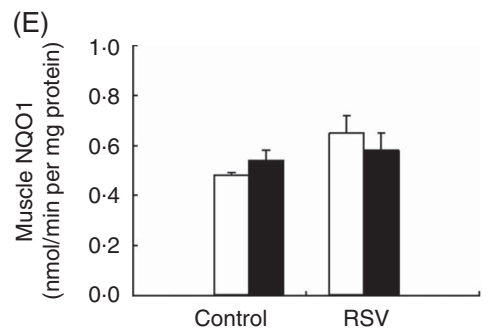

Fig. 5. Effect of resveratrol (RSV) and/or training on antioxidant activities of the gastrocnemius muscle in old mice. (A) Catalase (CAT), (B) superoxide dismutase (SOD), (C) glutathione peroxidise (GPx), (D) cytochrome $B_{5}$ reductase $\left(C_{1} B_{5} R a s e\right)$ and $(E) ~ N A D(P) H$ quinone dehydrogenase 1 (NQO1) activities measured in nmol/ min per mg protein. Values are means with their standard errors of different endogenous antioxidant activities in old animals. $\square$, Non-trained animals (sedentary);

口, trained animals. ${ }^{a}$ Significant difference $v$. control and non-trained group, $P<0 \cdot 05$, by using two-way ANOVA test.

\section{Discussion}

Our results demonstrate that a low daily intake of RSV primes the effect of exercise, increasing the performance in mature and particularly in old animals whereas no effects are shown in young animals. Importantly, the effect of RSV was obtained even when the animals started the supplementation with RSV at an advanced age. Thus, no lifelong treatment with RSV is needed to obtain a priming effect. Physical activity improvement was higher in old animals probably because of the higher 


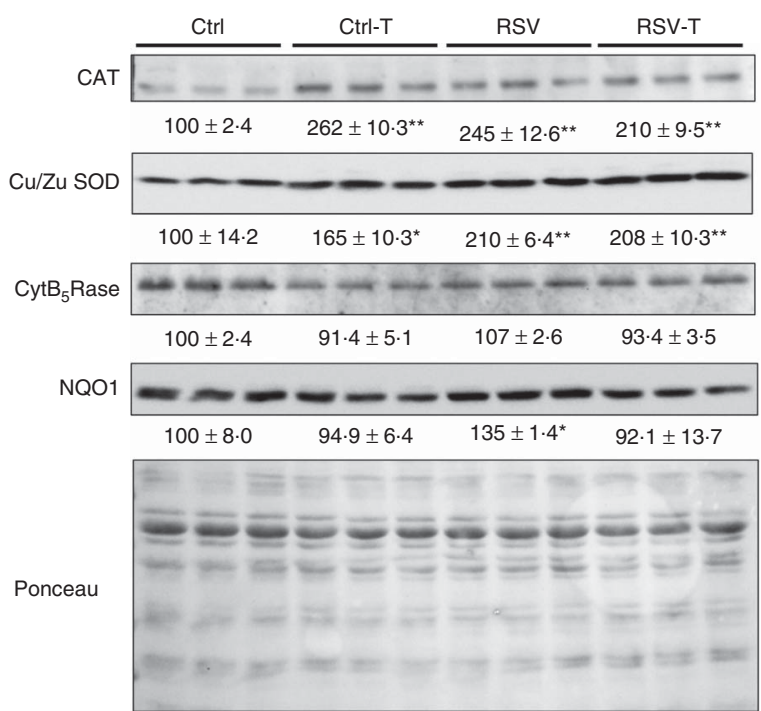

Fig. 6. Antioxidant protein levels in the gastrocnemius muscle of old animals fed resveratrol (RSV) and/or trained. Blots of catalase (CAT), $\mathrm{Cu} / \mathrm{Zn}$ superoxide dismutase (SOD), cytochrome $B_{5}$ reductase $\left(C_{1} \mathrm{~B}_{5}\right.$ Rase) and $\mathrm{NAD}(\mathrm{P}) \mathrm{H}$ quinone dehydrogenase 1 (NQO1) proteins determined by Western blotting. Ponceau, used as control loading, is also indicated. Levels indicated as the percentage of signal $v$. levels found in control group. Values are means with their standard errors of each group. * Significant difference $v$. control group, $P<0.05$; ** significant differences $v$. control group, $P<0.01$ by using one-way ANOVA test.

protection against oxidative damage and the increase in mitochondrial mass in muscle found in these animals.

Our results are in agreement with a recent study performed in rats supplemented with a similar dose of RSV and exercised through swimming, where the combination of RSV and exercise increased the effect on the phosphatidylinositol-4,5-bisphosphate 3-kinase/protein kinase B/forkhead box O (PI3K/AKT/FOXO) pathway, on the reduction of TNF- $\alpha$ and apoptotic markers in the hearts of 18-month-old rats ${ }^{(30)}$. The age-dependent effect agrees with previous studies performed in mice fed under every-otherday CR model, which showed the same age-dependent effect in relationship with the antioxidant protection ${ }^{(31)}$. This age-dependent effect has also been found in mice and rat fed under classical CR with higher effect in old than in young animals ${ }^{(32,33)}$. Old animals suffered an increase in lipid peroxidation in comparison with young and mature animals and RSV was able to decrease these levels. This suggests that in conditions where oxidative stress occurs, RSV produces benefits probably by increasing the activity of endogenous antioxidants. Interestingly, we found the same result in previous studies using CR as a prolongevity effector ${ }^{(32,33)}$. Old mice and rats under $\mathrm{CR}$ showed changes in antioxidant protection in liver plasma membrane, whereas young animals did not respond by modulating CytB5Rase and NQO1 activities and levels ${ }^{(32,33)}$. The same effect was also found in the brain ${ }^{(34)}$. Interestingly, in humans, we have also found an age-dependent response to exercise in the protection against lipid peroxidation in blood plasma. Young individuals show low levels of lipid peroxidation in plasma in comparison with old people. Exercise did not produce changes in young people, whereas we found a direct relationship between the level of physical activity and coenzyme $\mathrm{Q}_{10}$ levels in plasma that is involved in the protection against oxidative damage of lipoproteins. In fact, higher physical activity only in old people increased coenzyme $\mathrm{Q}_{10}$ levels in plasma and decreased lipid peroxidation and LDL oxidation ${ }^{(35,36)}$. It seems that age is an important factor in the induction of antioxidant response by RSV, CR or exercise probably because of more delicate antioxidant equilibrium during ageing.

Several studies have indicated that polyphenols are considered antioxidants, but the clearest effects are that they modulate endogenous mechanisms against oxidative stress instead of acting directly as antioxidants ${ }^{(37)}$. This action is exerted by increasing the activity of endogenous antioxidant enzymes such as SOD, CAT, GPx and others and also by decreasing the activity of enzymes that produce reactive oxygen species in cells such as NADPH-oxidase or hypoxanthine/xanthine oxidase ${ }^{(38)}$. In agreement with these studies, we have also recently found that RSV modulates liver antioxidant activities in old animals ${ }^{(23)}$, in an effect that can be related with lower inflammation in this organ $^{(39)}$. Interestingly, the effect of RSV and also physical activity was organ dependent, being more clear in the liver, muscle and heart but less effective in the kidney and brain ${ }^{(40)}$.

Bioactive compounds such as polyphenols, CR and exercise also induce molecular mechanisms involved in mitochondrial biogenesis and turnover, avoiding the accumulation of damaged mitochondria in tissues, the increase in reactive oxygen species levels and accumulation of dysfunctional mitochondria $^{(41)}$. In fact, the accumulation of abnormalities in the mitochondrial electron transport chain has been associated with muscle fibre loss and sarcopenia in rats ${ }^{(42)}$. Recently, in an accelerated model of ageing, it has been shown that sarcopenia is affected by dysregulation of the control of mitochondrial quality ${ }^{(43)}$. Exercise has been proposed to modulate the activity of the mitochondrial components affecting the development of sarcopenia, supporting the mitochondrial theory of ageing ${ }^{(44)}$. Regulators of mitochondrial physiology and biogenesis such as PGC- $1 \alpha$ that are induced by exercise or RSV have been associated with the prevention of loss of skeletal muscle mass and strength during ageing ${ }^{(45)}$. Taking these evidences into consideration, we can believe that bioactive compounds, CR and exercise can contribute to maintain a higher activity of organs and tissues during ageing and, in the case of skeletal muscle, to delay the reduction of physical capacity and age-related sarcopenia. Our results indicate that RSV in combination with exercise increases the amount of mitochondria and probably its capacity. The higher performance found in trained animals supplemented with RSV can be due to a higher capacity to increase fat oxidation as has been demonstrated with CR or RSV in high-fat-fed animals ${ }^{(10,11,46)}$. It is known that RSV increases fat metabolism in muscle ${ }^{(11,46)}$, enhancing fatty acid oxidation and improving mitochondrial efficiency ${ }^{(47)}$. Moreover, RSV could be considered as an ergogenic compound improving muscle response to exercise and probably protecting muscle against weakness and sarcopenia during ageing.

Interestingly, it seems that RSV mimics the effects we have found with CR in muscle. In a previous study, we found that CR was able to increase physical capacity in young mice ${ }^{(29)}$. This effect has been related to a higher mitochondrial capacity in the gastrocnemius muscle determined by the increase in fatconsuming type I fibres and a better architecture of interfibrillar 
(A)

Muscle cytochrome C
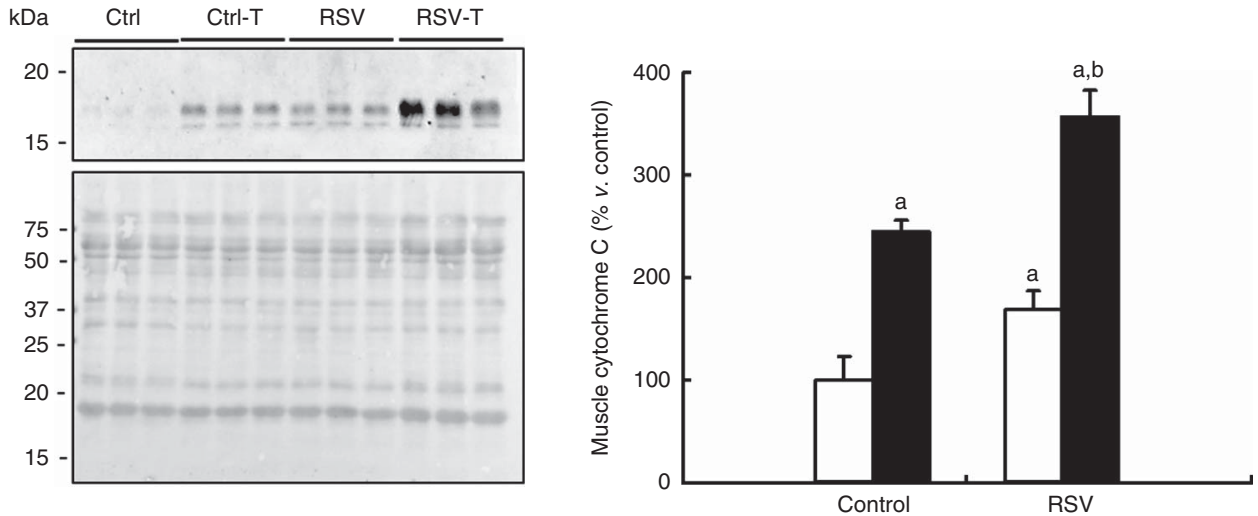

(B)

Muscle TFAM
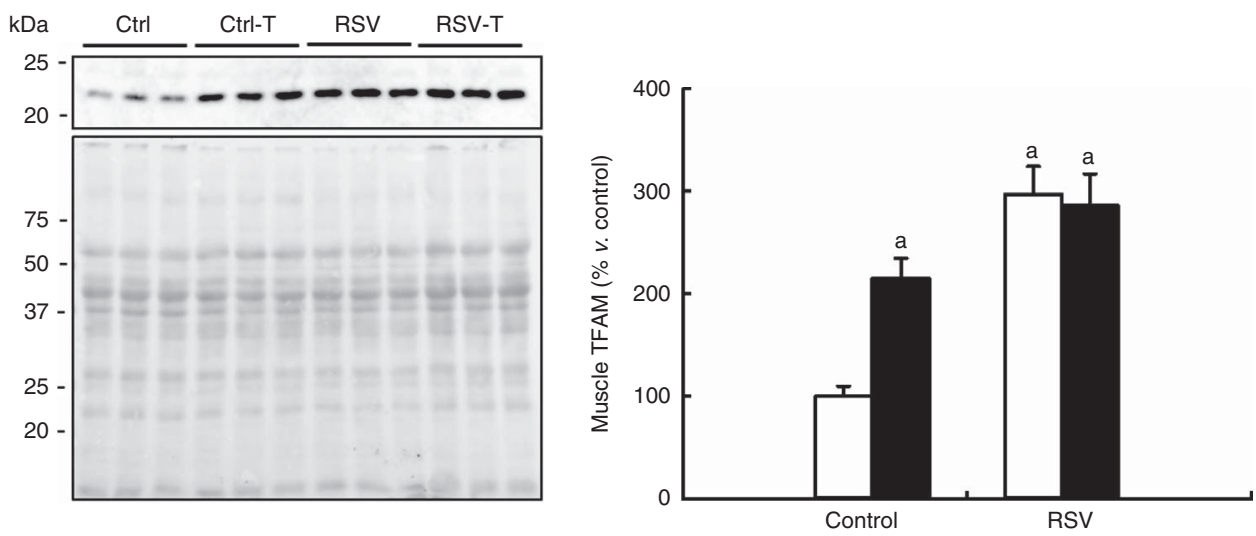

(C)

Muscle NRF1
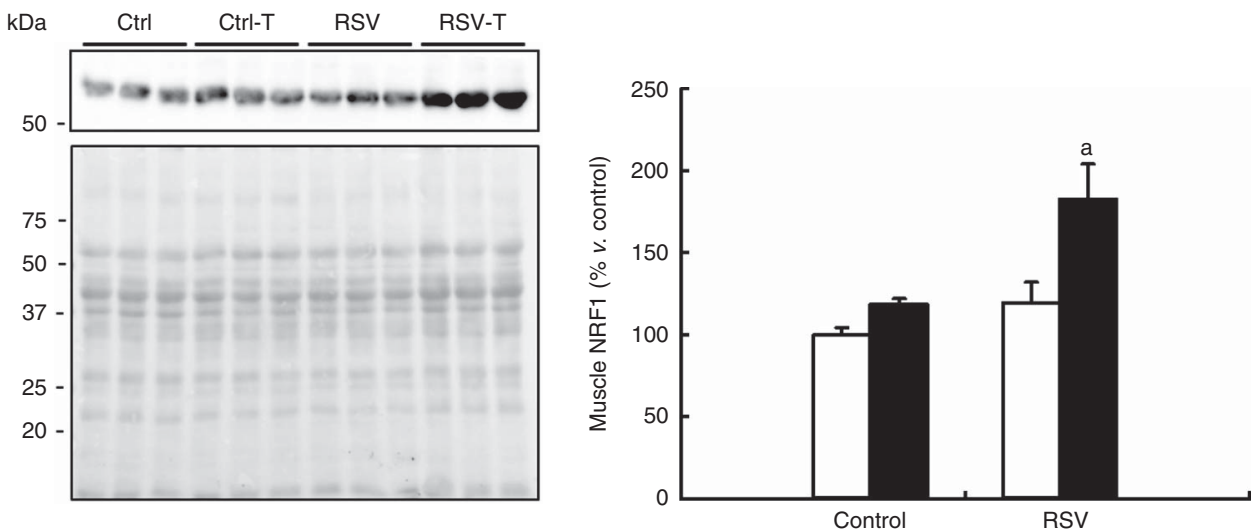

Fig. 7. Determination of mitochondrial mass markers in the gastrocnemius muscle of old mice. Western blotting of cytochrome $C$ (A), TFAM (B), anti-nuclear respiratory factor-1 (NRF1) (C) and respective Ponceau S staining and densitometry quantification of blots. Quantification is represented as the percentage $v$. control group in mean values and standard deviations. ${ }^{a}$ Significant difference $v$. control group, $P<0.05$; ${ }^{b}$ significant differences $v$. resveratrol (RSV)-treated and non-trained group, $P<0.05$, by using two-way ANOVA test. $\square$, Sedentary; $\square$, trained.

mitochondria, increasing the number and modifying their shape in this muscle ${ }^{(29)}$. The same effect has been found in old animals in which the combination of $\mathrm{CR}$ and exercise increased the performance of animals on the treadmill (Rodriguez-Bies, unpublished results). Muscle of old mice under CR and exercise also showed a higher number of type I fibres. This type of fibres was also increased by RSV in mice fed high-fat diets ${ }^{(10)}$.
The most controversial problem with the effect of bioactive compounds is to determine whether the positive effects found in preclinical studies performed in model animals produce the same response in humans. Regarding the effect of different polyphenols on physical capacity in humans, different clinical trials carried out to date have been unsuccessful or show controversial results and further studies are needed. 
The amount of polyphenols, the duration of the supplementation and the type of exercise are important factors that affect the results. Taking into consideration the low dose used in our study, in humans, a dairy intake amount of $1200 \mathrm{mg} /$ person (approximately $75 \mathrm{~kg}$ ) would be needed. However, many of the doses used in humans are not higher than 500-600 mg/d.

A recent study suggested a RSV-dependent impairment in the effect of exercise in old men ${ }^{(20)}$. These authors showed that the use of RSV in old men blunted the increase in maximal oxygen uptake found after training. On the other hand, another study of the same group showed that RSV inhibited the effect of exercise on protein carbonylation and TNF- $\alpha$ mRNA decrease in old skeletal muscle ${ }^{(22)}$. However, in these studies, the changes induced by exercise were minor and affected only a few variables of the total, and, in many of the cases, RSV and placebo showed the same effect as has been highlighted by other authors $^{(48,49)}$. In another recent study, a low dose of RSV $(100 \mathrm{mg} / \mathrm{d})$ during $90 \mathrm{~d}$ in young military firefighters did not produce significant effects on antioxidant capacity against strenuous exercise but, contrary to the findings in old people ${ }^{(22)}$, RSV did reduce the levels of proinflammatory cytokines $^{(21)}$. Furthermore, it has been shown that combination of RSV and quercetin in young athletes reduces the oxidation of lipids after exercise by mechanisms that are not related with their molecular antioxidant capacity ${ }^{(50)}$. Another recent study performed with a moderate dose of RSV (500 mg) combined with $10 \mathrm{mg}$ of piperine, an alkaloid found in black and long pepper, found improvement in mitochondrial capacity after light intense exercise in healthy young individuals ${ }^{(51)}$. Furthermore, a RSV dose of $600 \mathrm{mg} / \mathrm{d}$ for $7 \mathrm{~d}$ did not produce deleterious effects or positive effects in mature marathon runners (40-55 years), although a light trend to decrease inflammatory markers in plasma such as C-reactive protein and muscle soreness was reported ${ }^{(52)}$

As has been previously indicated, the different results found in studies regarding the use of different nutraceuticals in sport performance in humans indicate the need for more research on the effect of these compounds in humans ${ }^{(53)}$. Probably human studies need to fix the correct dose of RSV and other polyphenols and the time of supplementation to determine the positive or negative effects of these compounds and the effect of different combinations of different polyphenols on human physiology. Our study suggests that the age of the organism is another important factor and indicates that the effect of these compounds can be more relevant in aged than in mature or young organisms. Whether this age-dependent effect can be extrapolated to humans remains to be determined. Probably higher doses of RSV or combinations with other polyphenols will produce better results. RSV can be a promising dietary supplement, which can enhance the physical capacity of elderly people and avoid to some degree age-dependent sarcopenia, permitting a more active and healthy ageing. Probably, a higher consumption of fresh and polyphenol-rich vegetables and other bioactive compounds that modulate mitochondrial activity in muscle could increase the efficiency of physical exercise even in the elderly, thereby increasing healthspan and delaying frailty.

\section{Acknowledgements}

The authors thank Rosario Rodríguez Griñolo for her advice in statistics.

The research group is financed by the Andalusian Government as BIO177 group through FEDER funds (European Commission). Research has been financed by the Spanish Government grant DEP2012-39985 (Spanish Ministry of Science and Innovation). T. B. T. received a fellowship from the AECID program (Spanish Ministry of Foreign Affairs). E. R.-B., P. N. and G. L.-L. are also members of the Centro de Investigación Biomédica en Red de Enfermedades Raras, Instituto Carlos III.

G. L.-L.: design, conduct, analysis and writing of the article; E. R.-B. and T. B. T.: development of the research and analysis of data; P. N.: analysis of the results and writing of the article.

The authors declare that there are no conflicts of interest.

\section{References}

1. Evans WJ \& Campbell WW (1993) Sarcopenia and age-related changes in body composition and functional capacity. $J$ Nutr 123, 465-468.

2. Landi F, Liperoti R, Fusco D, et al. (2012) Prevalence and risk factors of sarcopenia among nursing home older residents. J Gerontol A Biol Sci Med Sci 67, 48-55.

3. Sanchis-Gomar F, Pareja-Galeano H, Mayero S, et al. (2014) New molecular targets and lifestyle interventions to delay aging sarcopenia. Front Aging Neurosci 6, 156.

4. Lopez-Lluch G \& Navas P (2015) Calorie restriction as an intervention in ageing. J Physiol 594, 2043-2060.

5. Zhao H, Li X, Li N, et al. (2014) Long-term resveratrol treatment prevents ovariectomy-induced osteopenia in rats without hyperplastic effects on the uterus. Br J Nutr 111, 836-846.

6. Miranda J, Portillo MP, Madrid JA, et al. (2013) Effects of resveratrol on changes induced by high-fat feeding on clock genes in rats. Br J Nutr 110, 1421-1428.

7. Shukitt-Hale B, Bielinski DF, Lau FC, et al. (2015) The beneficial effects of berries on cognition, motor behaviour and neuronal function in ageing. Br J Nutr 114, 1542-1549.

8. Kulkarni SS \& Canto C (2015) The molecular targets of resveratrol. Biochim Biophys Acta 1852, 1114-1123.

9. Timmers S, Konings E, Bilet L, et al. (2011) Calorie restrictionlike effects of 30 days of resveratrol supplementation on energy metabolism and metabolic profile in obese humans. Cell Metab 14, 612-622.

10. Lagouge M, Argmann C, Gerhart-Hines Z, et al. (2006) Resveratrol improves mitochondrial function and protects against metabolic disease by activating SIRT1 and PGC-1alpha. Cell 127, 1109-1122.

11. Baur JA, Pearson KJ, Price NL, et al. (2006) Resveratrol improves health and survival of mice on a high-calorie diet. Nature 444, 337-342.

12. Hood DA (2009) Mechanisms of exercise-induced mitochondrial biogenesis in skeletal muscle. Appl Physiol Nutr Metab 34, 465-472.

13. Jager S, Handschin C, St-Pierre J, et al. (2007) AMP-activated protein kinase (AMPK) action in skeletal muscle via direct phosphorylation of PGC-1alpha. Proc Natl Acad Sci US A 104, 12017-12022.

14. Winder WW, Holmes BF, Rubink DS, et al. (2000) Activation of AMP-activated protein kinase increases mitochondrial enzymes in skeletal muscle. J Appl Physiol (1985) 88, 2219-2226.

15. Dolinsky VW, Jones KE, Sidhu RS, et al. (2012) Improvements in skeletal muscle strength and cardiac function induced by 
resveratrol during exercise training contribute to enhanced exercise performance in rats. J Physiol 590, 2783-2799.

16. Menzies KJ, Singh K, Saleem A, et al. (2013) Sirtuin 1-mediated effects of exercise and resveratrol on mitochondrial biogenesis. J Biol Chem 288, 6968-6979.

17. Murase T, Haramizu S, Ota N, et al. (2009) Suppression of the aging-associated decline in physical performance by a combination of resveratrol intake and habitual exercise in senescence-accelerated mice. Biogerontology 10, 423-434.

18. Hart N, Sarga L, Csende Z, et al. (2013) Resveratrol enhances exercise training responses in rats selectively bred for high running performance. Food Chem Toxicol 61, 53-59.

19. Craig DM, Ashcroft SP, Belew MY, et al. (2015) Utilizing small nutrient compounds as enhancers of exercise-induced mitochondrial biogenesis. Front Physiol 6, 296.

20. Gliemann L, Schmidt JF, Olesen J, et al. (2013) Resveratrol blunts the positive effects of exercise training on cardiovascular health in aged men. $J$ Physiol 591, 5047-5059.

21. Macedo RC, Vieira A, Marin DP, et al. (2015) Effects of chronic resveratrol supplementation in military firefighters undergo a physical fitness test - a placebo-controlled, double blind study. Chem Biol Interact 227, 89-95.

22. Olesen J, Gliemann L, Bienso R, et al. (2014) Exercise training, but not resveratrol, improves metabolic and inflammatory status in skeletal muscle of aged men. J Physiol 592, 1873-1886.

23. Tung BT, Rodriguez-Bies E, Ballesteros-Simarro $\mathrm{M}$, et al. (2014) Modulation of endogenous antioxidant activity by resveratrol and exercise in mouse liver is age dependent. I Gerontol A Biol Sci Med Sci 69, 398-409.

24. Aebi H (1984) Catalase in vitro. In Methods in Enzymology, vol. 105, pp. 121-126 [P Lester, editor]. Amsterdam, The Netherlands: Academic Press.

25. Flohé L \& Günzler WA (1984) Assays of glutathione peroxidase. In Methods in Enzymology, vol. 105, pp. 114-120 [P Lester, editor]. Amsterdam, The Netherlands: Academic Press.

26. Benson AM, Hunkeler MJ \& Talalay P (1980) Increase of NAD (P)H:quinone reductase by dietary antioxidants: possible role in protection against carcinogenesis and toxicity. Proc Natl Acad Sci U S A 77, 5216-5220.

27. Bello RI, Alcain FJ, Gomez-Diaz C, et al. (2003) Hydrogen peroxide- and cell-density-regulated expression of $\mathrm{NADH}-$ cytochrome b5 reductase in HeLa cells. J Bioenerg Biomembr 35, 169-179.

28. Romero-Calvo I, Ocon B, Martinez-Moya P, et al. (2010) Reversible Ponceau staining as a loading control alternative to actin in Western blots. Anal Biochem 401, 318-320.

29. Rodriguez-Bies E, Santa-Cruz Calvo S, Fontan-Lozano A, et al. (2010) Muscle physiology changes induced by every other day feeding and endurance exercise in mice: effects on physical performance. PLOS ONE 5, e13900.

30. Lin CH, Lin CC, Ting WJ, et al. (2014) Resveratrol enhanced FOXO3 phosphorylation via synergetic activation of SIRT1 and PI3K/Akt signaling to improve the effects of exercise in elderly rat hearts. Age 36, 9705.

31. Rodriguez-Bies E, Navas P \& Lopez-Lluch G (2015) Agedependent effect of every-other-day feeding and aerobic exercise in ubiquinone levels and related antioxidant activities in mice muscle. J Gerontol A Biol Sci Med Sci 70, 33-43.

32. Lopez-Lluch G, Rios M, Lane MA, et al. (2005) Mouse liver plasma membrane redox system activity is altered by aging and modulated by calorie restriction. Age 27, 153-160.

33. De Cabo R, Cabello R, Rios M, et al. (2004) Calorie restriction attenuates age-related alterations in the plasma membrane antioxidant system in rat liver. Exp Gerontol 39, 297-304.

34. Hyun DH, Emerson SS, Jo DG, et al. (2006) Calorie restriction up-regulates the plasma membrane redox system in brain cells and suppresses oxidative stress during aging. Proc Natl Acad Sci U S A 103, 19908-19912.

35. Del Pozo-Cruz J, Rodriguez-Bies E, Ballesteros-Simarro M, et al. (2014) Physical activity affects plasma coenzyme Q10 levels differently in young and old humans. Biogerontology 15, 199-211.

36. Del Pozo-Cruz J, Rodriguez-Bies E, Navas-Enamorado I, et al. (2014) Relationship between functional capacity and body mass index with plasma coenzyme Q10 and oxidative damage in community-dwelling elderly-people. Exp Gerontol 52, 46-54.

37. Halliwell B, Rafter J \& Jenner A (2005) Health promotion by flavonoids, tocopherols, tocotrienols, and other phenols: direct or indirect effects? Antioxidant or not? Am J Clin Nutr 81, 268S-276S.

38. Carrizzo A, Forte M, Damato A, et al. (2013) Antioxidant effects of resveratrol in cardiovascular, cerebral and metabolic diseases. Food Chem Toxicol 61, 215-226.

39. Tung BT, Rodriguez-Bies E, Talero E, et al. (2015) Anti-inflammatory effect of resveratrol in old mice liver. Exp Gerontol 64, 1-7.

40. Tung BT, Rodriguez-Bies E, Thanh HN, et al. (2015) Organ and tissue-dependent effect of resveratrol and exercise on antioxidant defenses of old mice. Aging Clin Exp Res 27, 775-783.

41. Lopez-Lluch G, Santos-Ocana C, Sanchez-Alcazar JA, et al. (2015) Mitochondrial responsibility in ageing process: innocent, suspect or guilty. Biogerontology 16, 599-620.

42. Bua EA, McKiernan SH, Wanagat J, et al. (2002) Mitochondrial abnormalities are more frequent in muscles undergoing sarcopenia. J Appl Physiol (1985) 92, 2617-2624.

43. Joseph AM, Adhihetty PJ, Wawrzyniak NR, et al. (2013) Dysregulation of mitochondrial quality control processes contribute to sarcopenia in a mouse model of premature aging. PLOS ONE 8, e69327.

44. Johnston AP, De Lisio M \& Parise G (2008) Resistance training, sarcopenia, and the mitochondrial theory of aging. Appl Physiol Nutr Metab 33, 191-199.

45. Wenz T, Rossi SG, Rotundo RL, et al. (2009) Increased muscle PGC-1alpha expression protects from sarcopenia and metabolic disease during aging. Proc Natl Acad Sci US A 106, 20405-20410.

46. Lopez-Lluch G, Irusta PM, Navas P, et al. (2008) Mitochondrial biogenesis and healthy aging. Exp Gerontol 43, 813-819.

47. Chen LL, Zhang HH, Zheng J, et al. (2011) Resveratrol attenuates high-fat diet-induced insulin resistance by influencing skeletal muscle lipid transport and subsarcolemmal mitochondrial beta-oxidation. Metabolism 60, 1598-1609.

48. Smoliga JM \& Blanchard OL (2013) Recent data do not provide evidence that resveratrol causes 'mainly negative' or 'adverse' effects on exercise training in humans. J Physiol 591, 5251-5252.

49. Buford TW \& Anton SD (2014) Resveratrol as a supplement to exercise training: friend or foe? J Physiol 592, 551-552.

50. McAnulty LS, Miller LE, Hosick PA, et al. (2013) Effect of resveratrol and quercetin supplementation on redox status and inflammation after exercise. Appl Physiol Nutr Metab 38, 760-765

51. Polley KR, Jenkins N, O'Connor P, et al. (2016) Influence of exercise training with resveratrol supplementation on skeletal muscle mitochondrial capacity. Appl Physiol Nutr Metab 41, 26-32.

52. Laupheimer MW, Perry M, Benton S, et al. (2014) Resveratrol exerts no effect on inflammatory response and delayed onset muscle soreness after a marathon in male athletes: a randomised, double-blind, placebo-controlled pilot feasibility study. Transl Med UniSa 10, 38-42.

53. Braakhuis AJ \& Hopkins WG (2015) Impact of dietary antioxidants on sport performance: a review. Sports Med $\mathbf{4 5}$, 939-955. 